Rabaska

Revue d'ethnologie de l'Amérique française

\title{
Marius Barbeau. Charlevoix, été 1916
}

\section{Serge Gauthier}

Volume 13, 2015

Présence de Marius Barbeau : l'invention du terrain en Amérique française. Autour d'un legs centenaire (1914-2014)

URI : https://id.erudit.org/iderudit/1033744ar

DOI : https://doi.org/10.7202/1033744ar

Aller au sommaire du numéro

Éditeur(s)

Société québécoise d'ethnologie

ISSN

1703-7433 (imprimé)

1916-7350 (numérique)

Découvrir la revue

Citer cet article

Gauthier, S. (2015). Marius Barbeau. Charlevoix, été 1916. Rabaska, 13, 16-21. https://doi.org/10.7202/1033744ar

Ce document est protégé par la loi sur le droit d'auteur. L'utilisation des services d'Érudit (y compris la reproduction) est assujettie à sa politique d'utilisation que vous pouvez consulter en ligne.

https://apropos.erudit.org/fr/usagers/politique-dutilisation/ 


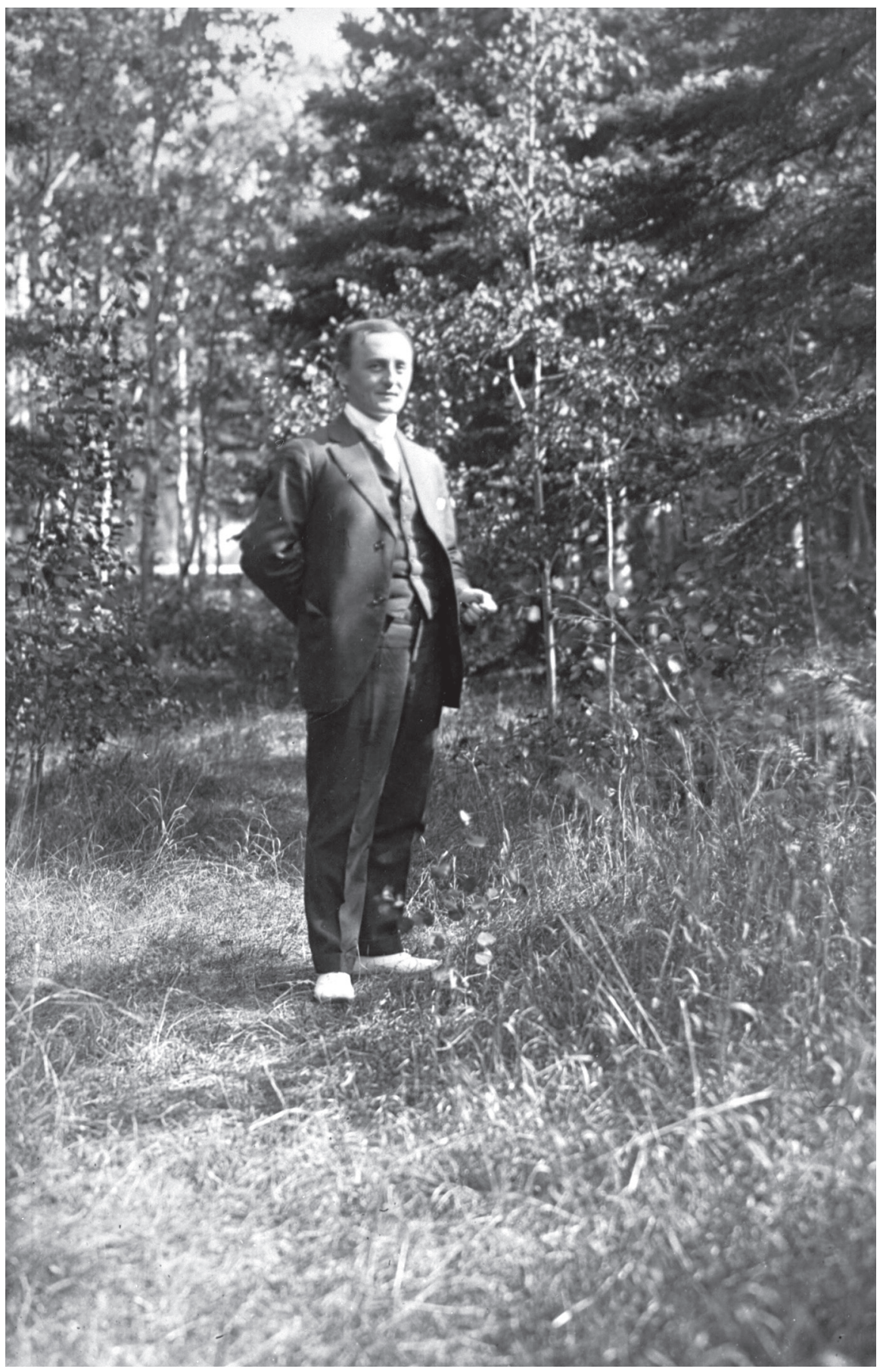

Marius Barbeau à Tadoussac, Québec, 1916.

Source : Musée canadien de l'histoire nº 37274. 


\title{
Marius Barbeau. Charlevoix, été 1916
}

\author{
Serge Gauthier \\ Société d'histoire de Charlevoix \\ Centre de recherche sur l'histoire et le patrimoine de Charlevoix
}

\section{«Il était une fois... » Charlevoix}

Dans l'esprit de Marius Barbeau retenons que la région de Charlevoix est propice aux contes et aux légendes et disons donc : «Il était une fois ».

Il était une fois d'abord une région, un territoire qui fut heurté il y a plus de 350 millions d'années par une immense météorite et que ce grand fracas a peut-être favorisé en ce lieu une sensibilité sismique sur laquelle nous reviendrons...

Un lieu appelé depuis 1855 du nom de Charlevoix ${ }^{1}$, d'abord pour désigner la circonscription électorale rattachée à ce vaste territoire. Et ce en l'honneur de Pierre-François-Xavier de Charlevoix (1682-1761), père jésuite et historien de la Nouvelle-France. Et nous, de la Société d'histoire de Charlevoix - qui célèbre cette année trente ans d'existence -, nous sommes fiers que notre région porte le nom de ce grand personnage.

Toutefois, le découvreur historique de notre territoire fut sans doute Jacques Cartier qui aborda l'île aux Coudres le 5 septembre 1535 et y trouva d'excellentes « noisilles » en plus " d'y ouir la messe » qui fut longtemps considérée comme la première célébrée en Amérique du Nord.

Au XVII siècle, Samuel de Champlain ne fut pas en reste puisqu'il nomma sur le territoire de nombreux sites et lieux et leur donna des appellations parfois très inquiétantes : «Rivière du Gouffre », « Malbaie », « Cap à l’Aigle », «Baie des Rochers ». Et quelquefois aussi plus invitantes : « Cap aux Oyes », «Petite Rivière », « Port au Persil ».

Le territoire fut habité en partie à cause d'un immense « tremble-terre » ou tremblement de terre survenu en 1663. Le toponyme «Les Éboulements » résulte de cet événement devenu presque fantastique sous la plume imagée

1. Sur l'histoire de la région de Charlevoix, $c f$. Serge Gauthier et Normand Perron, Charlevoix, histoire en bref, Québec, Presses de l'Université Laval, 2002 ; Normand Perron et Serge Gauthier, Histoire de Charlevoix, Québec, Presses de l'Université Laval-IQRC, « Histoire des régions du Québec » $14,2000,395 \mathrm{p}$. 
des pères jésuites. L'intendant Jean Talon crut y déceler une possibilité de développement économique et il fit chercher une improbable mine de fer dont le potentiel s'avéra décevant, mais cela amena ensuite le peuplement sédentaire de notre région à partir de 1675 . Le premier résident officiel fut Claude Bouchard, un habitant de la Petite-Rivière-Saint-François.

À titre informatif, des tremblements de terre importants ou dits historiques sont survenus dans Charlevoix en 1663, 1791, 1860, 1870, 1925, mais, au fond, outre la crainte provisoire de ces séismes au sein de la population, rien de bien inquiétant ne se produisit suite à ces événements en principe fort dramatiques ${ }^{2}$.

Claude Bouchard fut-il vraiment le premier habitant de Charlevoix ? Pas vraiment puisque un squatter du nom de Saint Aubin vint s'établir auparavant (vers 1666) pour se cacher du fait qu'il avait tué l'amant de sa femme à l'île d'Orléans. M ${ }^{\text {gr }}$ de Laval, évêque de Québec et propriétaire des terres du Séminaire où se trouve alors la Baie-Saint-Paul, le fit chasser sans hésiter. De nos jours, la polyvalente de Baie-Saint-Paul porte le nom de ce personnage qui fut quand même un meurtrier.

Des personnages légendaires, il en est apparu plusieurs dans notre région. Parlons de l'homme fort Jean-Baptiste Grenon de Baie-Saint-Paul : cet homme, qui se défendit sans difficulté contre un ours, arrachait sans peine toutes les souches d'arbre qui se présentaient à lui et, bien qu'appréhendé par les Anglais en 1759, il fut libéré par eux, car ces derniers furent incapables de le maîtriser pour cause de trop grande force. La fille de Grenon, Marie, portait sur sa tête un minot de sel et marchait allégrement. À La Malbaie, l'homme fort Davi Archange (David Bouchard) écrasait une pomme de terre de sa main, sur laquelle il faisait danser sa femme toute menue, mais pesant quand même deux cents livres... L'homme fort du Saguenay, Victor Delamarre, eut peur de lui et préféra retourner au Saguenay plutôt que de l'affronter.

Il y avait aussi le père de La Brosse, jésuite mystérieux qui avait prédit sa mort par avance, Alexis Lapointe dit le Trotteur courant plus vite qu'un cheval, Boily le Ramancheur défaisant des chats pour montrer son habileté, et combien d'autres faits et personnages étranges qui se trouvaient un peu retirés derrière nos montagnes, nos caps élevés si imposants. Et il a fallu Marius Barbeau pour les mettre en lumière. Il devint ainsi le véritable découvreur folklorique de Charlevoix.

\section{« Il était donc une fois... » et Marius Barbeau vint en Charlevoix}

Mais quand Barbeau est-il venu en Charlevoix pour la première fois ? Du moins, à titre de chercheur de folklore, ce fut à l'été 1916. Alors que faisait

2. Christian Harvey, Les Tremblements de terre dans Charlevoix selon les documents historiques. Effets sur les bâtiments et les terrains, La Malbaie, Éditions Charlevoix, 2015, 123 p. 
rage la Première Guerre mondiale en Europe, tout paraissait très paisible dans Charlevoix. On pourrait dire que c'était un jeune chercheur, mais il avait quand même 33 ans. Il travaillait déjà sur le terrain à titre d'anthropologue depuis 1911 pour le Musée national du Canada.

Mais comment Marius Barbeau s'est-il intéressé à Charlevoix comme territoire folklorique ? Il y a sur ce sujet bien des légendes, une sorte de folklore du terrain folklorique et Barbeau était lui-même un grand conteur. Laissons-nous donc charmer par sa légende pour un moment.

Ainsi, Marius Barbeau, en quête de contes français à la demande du grand anthropologue américain Franz Boas, s'est d'abord rendu sur la réserve de Lorette, près de Québec. Il trouva bien de ces contes français, mais un autochtone lui affirma bientôt que « nulle part mieux que derrière les montagnes du Cap-Tourmente, il trouverait des contes français », soit auprès des habitants de cette région de Charlevoix perçue alors comme isolée. Et puis disait l'autochtone, ces gens-là possèdent « un excellent rhum de la Jamaïque », ce qui ne devrait pas faire de tort pour la cueillette de folklore...

Marius Barbeau suivit le conseil de l'autochtone et il se rendit dans Charlevoix pour la première fois à l'été 1916 deux années seulement après ses premières enquêtes auprès de francophones. Charlevoix est donc un des vieux terroirs de recherche pour Barbeau. Il fut l'un de ses plus féconds. Il séjourna ainsi trois mois dans la région à l'été 1916.

Mais comment Barbeau parvient-il, en 1916, à franchir les montagnes derrière le Cap-Tourmente, par-delà les imposants caps qui paraissaient isoler Charlevoix ? En auto ? Il n'y en avait pas à l'époque dans la région ! En train ? Ce lien ferroviaire ne se rendra à La Malbaie qu'en 1919 ! En calèche ? Ce parcours aurait été très aventureux et long par le chemin des caps. Par bateau, bien sûr, mais pas n'importe lequel : un bateau de la croisière du Saguenay et il s'agit du « Murray Bay». Barbeau devint-il donc un touriste comme un autre dans Charlevoix ? Sans doute pas, puisque sa présence n'allait pas passer inaperçue et qu'elle s'imposera dans l'histoire régionale, même nationale, et surtout dans le cheminement des études folkloriques ou ethnologiques de terrain au Québec, voire en Amérique du Nord.

Barbeau arriva donc au quai de Pointe-au-Pic où il vit dès sa sortie du bateau des autochtones (des Montagnais) qui vendaient de l'artisanat et notamment des canots d'écorce. Il monte sans trop tarder dans une calèche et elles sont nombreuses sur ce quai à cette époque. Sa destination : le village de Saint-Irénée. Barbeau n'est pas très intéressé à s'attarder à Pointe-au-Pic (Murray-Bay), lieu de villégiature très connu qui ne lui semble pas propice pour la quête de folklore.

Alors, il s'en va en calèche vers Saint-Irénée, mais le conducteur avertit Marius Barbeau : le voyage vers ce village ne sera pas simple et plutôt 


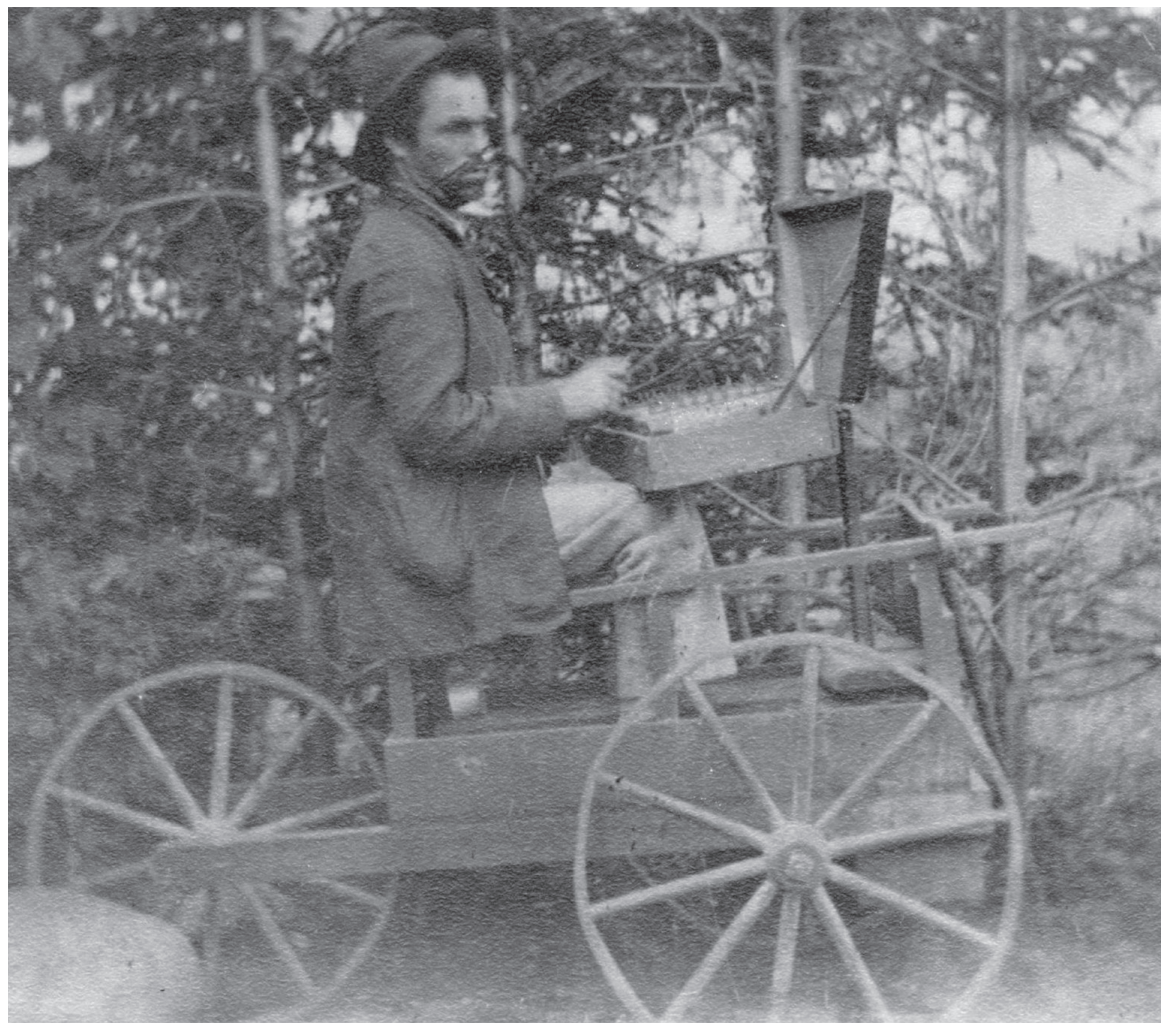

Louis Simard dit l'Aveugle jouant du tympanon, vers 1900.

Copie d'une photographie originale sur zinc provenant de

$\mathrm{M}^{\text {me }}$ Fidèle Dufour de Sault-au-Mouton.

Source : Musée canadien de l'histoire, nº PR2003-025.

cahoteux ${ }^{3}$. Sur un chemin de terre sinueux, il faut alors monter et redescendre d'abruptes côtes et tout cela est fort exigeant et très poussiéreux. Barbeau, plutôt surpris, encaissa bien le coup !

Il séjourna à l'hôtel local de Saint-Irénée qui existe sur le bord du fleuve, non loin du Domaine Gil'Mont ${ }^{4}$ habité en saison estivale par le millionnaire Rodolphe Forget. La question de savoir si Marius Barbeau s'est rendu ou non au Domaine Gil'Mont se pose sans doute ici et nous pouvons croire qu'il y est venu. Il pourrait même y avoir rencontré lors d'une soirée artistique un certain chanteur folklorique du nom de Louis Simard dit l'Aveugle...

Cet homme paraissait très impressionnant. Bien qu'aveugle, il circule sans difficulté sur la Haute-Côte-Nord, dans Charlevoix, au Saguenay, dans

3. Cf. Serge Gauthier, Marius Barbeau, le grand sourcier, Montréal, Éditions XYZ, 2001, 144 p.

4. Aujourd'hui connu sous le nom de Domaine Forget, lieu où s'est tenu ce colloque international consacré à Marius Barbeau. 
une simple charrette qu'il actionne avec la seule force de ses bras, durant toute la période estivale. Il ne perd jamais son chemin. Personne ne lui ferait de mal car Ti-Louis l'Aveugle, comme on dit, est un chanteur folklorique qui divertit la population avec ses chants et ses histoires. Il a dans sa tête des merveilles à chanter et à dire, il possède un ocarina, une « bioune » (sorte de cithare) et une petite tasse pour accepter les dons car l'Aveugle gagne sa vie ainsi comme ménestrel et troubadour ambulant.

Barbeau souhaite rapidement enregistrer ce personnage étonnant sur les cylindres de cire Edison qu'il a apportés avec lui. Mais ce dernier refuse, selon ce que raconte Barbeau. L'Aveugle doit d'abord se confesser puisque c'est alors la fête de la bonne Sainte-Anne (26 juillet), à l'église de Saint-Irénée, où il a été baptisé et jusque-là il ne peut rien chanter. Après ses dévotions, Louis l'Aveugle s'imposa comme un pittoresque et magistral informateur de Marius Barbeau. Il chanta notamment la longue complainte de Pyrame et Thisbée, ces amoureux babyloniens si romantiques et si malheureux...

Marius Barbeau est enchanté par la possibilité de trouver facilement des informateurs dans Charlevoix. Il a l'impression, selon ses dires, d'être dans une sorte « de pays des merveilles ». Au cours de l'été 1916, alors qu'il circule en calèche dans les rangs isolés de Charlevoix, Barbeau recueille surtout des contes et des chansons, mais aussi des éléments du langage populaire de Charlevoix. Barbeau va s'émerveiller de ce terrain folklorique unique et il reviendra souvent dans Charlevoix pour d'autres recherches, notamment dans les années 1920, 1930 et 1940, particulièrement chez ses amis Jean Palardy, dans la localité charlevoisienne de Saint-Urbain, ou l'écrivain et prêtre Félix-Antoine Savard, curé de la paroisse industrielle de Clermont.

Charlevoix est-il donc « un pays enchanté » pour Marius Barbeau? Nous n'aimons pas beaucoup cette notion de " pays enchanté » trop associée à l'histoire touristique de cette région pour la définir adéquatement. Mais un « pays enchanté » quand même, puisque les enquêtes effectuées à l'été 1916 dans Charlevoix permettent à Barbeau de rédiger un de ses premiers textes scientifiques significatifs : intitulé «Au pays des gourganes », en l'honneur de cette fève des marais dont la culture est si présente dans Charlevoix, il le présente en 1917 devant la Société royale du Canada. Disons donc simplement que Barbeau a beaucoup recueilli au cours de sa carrière, beaucoup écrit sur lui et sur son travail de terrain, à tel point qu'il faut parfois aller au-delà de sa légende personnelle pour retrouver les trésors qu'il nous a légués avec une plus grande justesse de point de vue. Voilà un beau défi de recherche, il me semble, à l'aube de ce colloque Marius-Barbeau. 